\title{
Changes in Effective Thermal Conductivity During the Carbothermic Reduction of Magnetite Using Graphite
}

\author{
SAEED KIAMEHR, HESHAM AHMED, NURNI VISWANATHAN, \\ and SESHADRI SEETHARAMAN
}

Knowledge of the effective thermal diffusivity changes of systems undergoing reactions where heat transfer plays an important role in the reaction kinetics is essential for process understanding and control. Carbothermic reduction process of magnetite containing composites is a typical example of such systems. The reduction process in this case is highly endothermic and hence, the overall rate of the reaction is greatly influenced by the heat transfer through composite compact. Using Laser-Flash method, the change of effective thermal diffusivity of magnetite-graphite composite pellet was monitored in the dynamic mode over a pre-defined thermal cycle (heating at the rate of $7 \mathrm{~K} / \mathrm{min}$ to $1423 \mathrm{~K}\left(1150^{\circ} \mathrm{C}\right)$, holding the sample for 270 minutes at this temperature and then cooling it down to the room temperature at the same rate as heating). These measurements were supplemented by Thermogravimetric Analysis under comparable experimental conditions as well as quenching tests of the samples in order to combine the impact of various factors such as sample dilatations and changes in apparent density on the progress of the reaction. The present results show that monitoring thermal diffusivity changes during the course of reduction would be a very useful tool in a total understanding of the underlying physicochemical phenomena. At the end, effort is made to estimate the apparent thermal conductivity values based on the measured thermal diffusivity and dilatations.

DOI: $10.1007 / \mathrm{s} 11663-017-0944-6$

(C) The Author(s) 2017. This article is published with open access at Springerlink.com

\section{INTRODUCTION}

RECENTLY, carbothermic reduction processes where carbonaceous material mixed with metallic oxide powder are heated to achieve reduction is being explored to supplement gas-based reduction processes. ${ }^{[1]}$ These reduction reactions are highly endothermic and hence, the overall rate of these reactions is greatly influenced by the heat transfer through powder mixture. Therefore, in such processes, the knowledge of the effective thermal conductivity of the composite pellet made of powder mixture is essential for process understanding and control. In addition, in such processes, the reaction is quite often accompanied by sample shrinkage, change of porosity, partial melting, and other physical changes apart from the chemical reaction. Traditional method to study gas-solid or solid-solid reactions accompanied by mass changes is thermogravimetry. While this method

SAEED KIAMEHR is with the Royal Institute of Technology (KTH), 10044 Stockholm, Sweden. HESHAM AHMED is with the Luleå Technical University, 97187 Luleå, Sweden and Central Metallurgical Research and Development Institute, P.O. Box 87Helwan, Cairo, Egypt. Contact e-mail: Hesham.ahmed@1tu.se NURNI VISWANATHAN is with the Luleå Technical University, and also with Indian Institute of Technology Bombay, 400076 Mumbai, India. SESHADRI SEETHARAMAN is with the Royal Institute of Technology (KTH), and also with Indian Institute of Technology Bombay.

Manuscript submitted April 27, 2016.

Article published online February 27, 2017. provides knowledge of the chemistry involved in the reaction by monitoring the mass change (due to loss of the gaseous product), it does not provide information regarding the physical changes and their impact on the overall reaction. For example, decrease of porosity will result in the decrease of the permeability of the product gases in the carbothermic reduction and consequently on the reaction rate. In situ measurement of thermal properties of the composite pellet also gives insights into the physicochemical changes occurring in the pellet during carbothermic reduction.

Attempts were made earlier to understand the physicochemical changes based on corresponding changes of thermal properties. Akiyama et al. ${ }^{2]}$ studied temperature dependency of thermal conductivity of iron ore agglomerates. Thermal conductivities of dense pure hematite, magnetite, wüstite, and metallic iron were measured as a function of temperature, and a linear relationship between thermal resistivity (reciprocal of thermal conductivity) and temperature was proposed. Further, the thermal conductivity of iron ore agglomerates from different origins has been measured by the same authors while being reduced to magnetite, wüstite and finally to metallic iron by $\mathrm{CO} / \mathrm{CO}_{2}$ or $\mathrm{H}_{2}$. It was found that the obtained thermal conductivity values for the processed iron ore agglomerates were remarkably lower than those of pure dense ones which of course could be attributed mainly to the existence of pores originating from oxygen removal. Expressions in literature $^{[2-6]}$ for effective thermal conductivity of iron ore 
agglomerates, that mainly consider porosity as the major parameter, were neither able to explain satisfactorily the measured values nor predict the difference between samples of identical composition and reduction state with different origin. Akiyama et al. ${ }^{[2]}$ then developed a modified unit cell, which was originally proposed by Luikov, ${ }^{[7]}$ wherein the solid sample was considered as having a structure composed of core and connecting parts. This modified unit cell model was found to explain the essential features of measured thermal conductivities of iron ore agglomerates. Moreover, it clearly identified the distinction of solid structure in samples reduced to the same degree but originated and prepared differently.

Thus, monitoring the changes in the effective thermal diffusivity that accompanies a reduction reaction will be extremely valuable in simulation and control of the process based on process fundamentals. However, systems where carbothermic reduction is considered are expected to be more complicated. To the present knowledge of the authors, estimation of the effective thermal conductivity of magnetite-graphite composite pellets has not been reported elsewhere. Therefore, the present investigation aims at evaluating the feasibility of the dynamic thermal conductivity measurements in order to follow the changes in this property of the composite pellet as the reaction proceeds. It was expected that this would enable a better understanding of the reaction kinetics and accompanying physicochemical changes.

\section{THEORETICAL BACKGROUND}

It is well-known that reduction reaction in a composite mixture containing carbon and iron oxide occurs through oxygen exchange in the gas phase. In the vicinity of the oxide particle, the carbon monoxide in the gas phase would reduce the oxide to form carbon dioxide. The carbon dioxide formed would then react with carbon particle to regenerate the $\mathrm{CO}$ back in the gas phase, effectively transferring oxygen and carbon from solid oxide and carbon phase to the gas phase as CO.

However, there is a need for an initiation mechanism so that sufficient amount of $\mathrm{CO}$ is produced to initiate the reduction and sustain the overall reaction. Thus, the mechanism of carbothermic reduction in carbon-iron oxide mixture can be described as follows ${ }^{[8]}$.

Stage I

$$
\begin{gathered}
\mathrm{C}(\mathrm{s})+0.5 \mathrm{O}_{2}(\mathrm{~g})=\mathrm{CO}(\mathrm{g}) \\
\mathrm{C}(\mathrm{s})+\mathrm{Fe}_{x} \mathrm{O}_{y}(\mathrm{~s})=\mathrm{Fe}_{x} \mathrm{O}_{y-1}(\mathrm{~s})+\mathrm{CO}(\mathrm{g})
\end{gathered}
$$

Stage II

$$
\begin{gathered}
\mathrm{Fe}_{x} \mathrm{O}_{y}(\mathrm{~s})+\mathrm{CO}(\mathrm{g})=\mathrm{Fe}_{x} \mathrm{O}_{y-1}(\mathrm{~s})+\mathrm{CO}_{2}(\mathrm{~g}) \\
\mathrm{C}(\mathrm{s})+\mathrm{CO}_{2}(\mathrm{~g})=2 \mathrm{CO}(\mathrm{g})
\end{gathered}
$$

in which $x=1,2,3$ and $y=1,3,4$ corresponding to wüstite, hematite or magnetite.

\section{Stage III}

$$
\begin{aligned}
& \mathrm{CO}(\mathrm{g}, \text { interior })=\mathrm{CO}(\mathrm{g}, \text { inert gas phase }) \\
& \mathrm{CO}_{2}(\mathrm{~g}, \text { interior })=\mathrm{CO}_{2}(\mathrm{~g}, \text { inert gas phase })
\end{aligned}
$$

The first stage is initiated through the residual air which is trapped within the oxide-carbon mixture. This oxygen might react with solid carbon to produce CO (Eq. [1]). However, under the present experimental conditions, this is not likely to be the case in view of the system being evacuated and flushed with argon before the start of the experiments. The reaction can also be initiated by solid-solid reaction between carbon and oxide at the contact points (Eq. [2]). In the subsequent stage, locally produced carbon monoxide will further react with iron oxide producing lower oxides and $\mathrm{CO}_{2}$ (Eq. [3]). The produced $\mathrm{CO}_{2}$ will further react with solid carbon to produce $\mathrm{CO}$ by Boudouard reaction (or gasification reaction) as shown in Eq. [4] which is highly endothermic. This reaction will further promote the reduction (Eq. [3]). The reaction will come to an end once the gaseous species are effectively removed (Eqs. [5, 6]). It can also be stopped if severe sintering removes the porosity in a way that product gas cannot diffuse out.

As stated previously, carbothermic reduction is an endothermic reaction. The rate of heat supply to the reactants and heat transport into the bulk bed play an important role in determination of the overall kinetics. This, in turn, is decided by thermal transport properties of the mixture. During carbothermic reduction in packed beds, since conduction is the dominant mechanism of heat transfer through the bulk bed, effective thermal conductivity is a crucial property. This quantity is a measure of how fast a material dissipates heat through conduction and is defined as the proportionality constant in Fourier's law of heat conduction. Thermal conductivity is related to thermal diffusivity according to Eq. [7] as follows:

$$
K=\alpha \rho C_{\mathrm{p}},
$$

where $K$ is the thermal conductivity $(\mathrm{W} / \mathrm{m} . \mathrm{K}), \alpha$ the thermal diffusivity $\left(\mathrm{m}^{2} / \mathrm{s}\right), \rho$ the density $\left(\mathrm{kg} / \mathrm{m}^{3}\right)$, and $C_{\mathrm{p}}$ the heat capacity $(\mathrm{J} / \mathrm{kg} \cdot \mathrm{K})$.

\section{EXPERIMENTAL}

\section{A. Materials and Samples Specifications}

Magnetite (Sigma-Aldrich, 99.99 pct trace metals basis) of $\sim 1 \mu \mathrm{m}$ particle size and graphite (Alfa-Aesar, synthetic, conducting grade, 99.9995 pct, -325 mesh) powders was chosen as reactants. The $\mathrm{C}$ : O molar ratio was kept at the stoichiometric ratio of 1 . After addition of $1 \mathrm{wt}$ pct binder (sodium bentonite, Alfa-Aesar), the reactants were mixed in an agate mortar.

Distilled water (as much as $\sim 25 \mathrm{wt}$ pct of the mixture) was added and after stirring, the mixture was divided 
into two groups of batches. The first one consisted of $\sim 200 \mathrm{mg}$ batches which would be used for the Thermogravimetric Analysis (TGA). The second group, being in $\sim 250 \mathrm{mg}$ batches, was retained for other tests. They were accordingly compacted under a pressure of $\sim 21 \mathrm{MPa}$ by means of a hydraulic press (Paul. Weber, Stgt-Uhlbach, Germany) and cylinder-piston steel moulds of $7.20 \mathrm{~mm}$ (for $200 \mathrm{mg}$ samples) and $12.15 \mathrm{~mm}$ internal diameter (for $250 \mathrm{mg}$ ones). Samples were dried by keeping them at room temperature for at least 24 hours. Thereafter, they were weighed in a microbalance and their thicknesses were measured using a micrometer. Thus, having the values of mass, thickness, and diameter, the initial apparent density was computed. Finally, the samples were examined by Scanning Electron Microscopy (SEM) to ensure that no microcracks were present in the samples.

\section{B. Measurement of Effective Thermal Diffusivity by the Laser-Flash Method}

A Laser-Flash unit (model TC-7000H/MELT, Sinku-Riko, Japan) with a maximum sample temperature limit of $1873 \mathrm{~K}\left(1600^{\circ} \mathrm{C}\right)$ was employed in the present study to measure the thermal diffusivity values in situ. A schematic diagram of the laser-flash unit is given in Figure 1.

In this method, the top side of a specimen is irradiated with a laser which provides an instantaneous energy

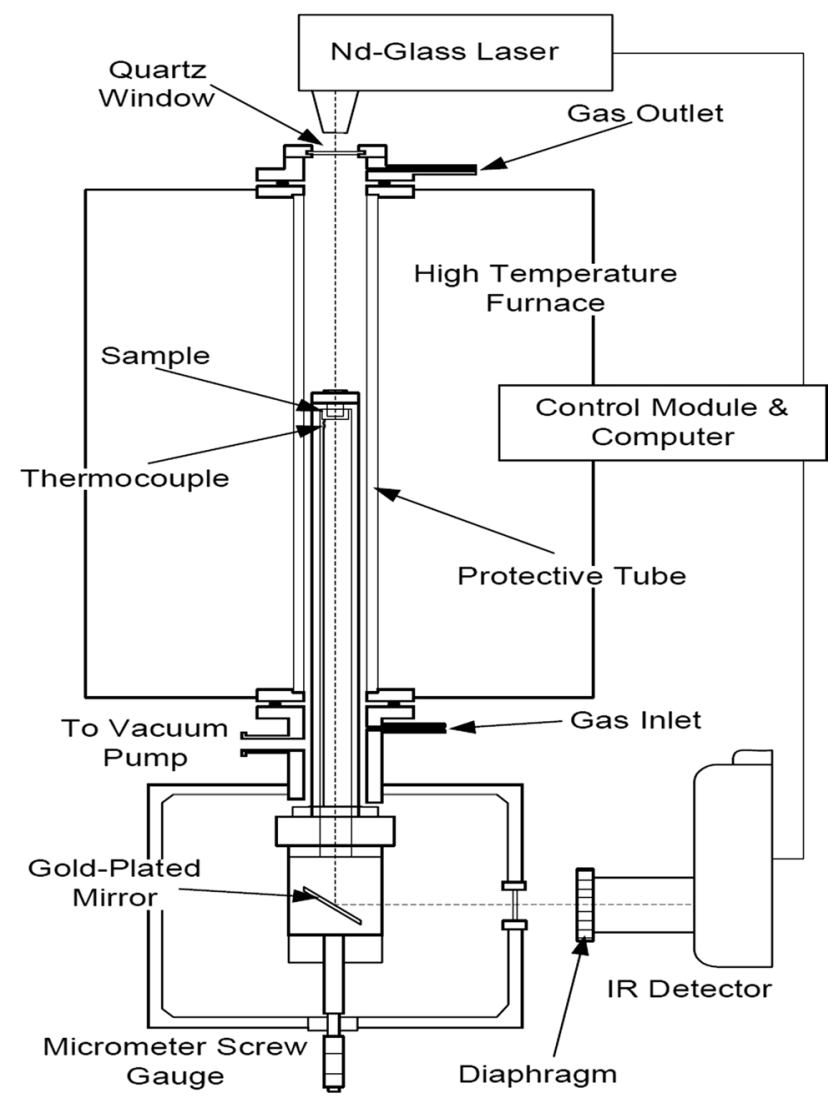

Fig. 1-Schematic diagram of the Laser-Flash unit, reprinted from Ref. [9]. pulse from a top mounted Nd-glass laser $(2.40 \mathrm{kV})$. The laser energy is absorbed by the top surface of the sample. The temperature of the rear face is monitored using a photovoltaic infra-red detector. The temperature rise of the back surface of the sample is plotted as a function of time. Figure 2 shows the principle of the laser-flash method.

The thermal diffusivity values were calculated based on Eq. [8], wherein $t_{1 / 2}$ is the time required for the rear surface to reach half of the maximum temperature. The value " 1.37 " is a factor calculated based on calibration measurements using standard stainless steel samples with different thicknesses, supplied by Sinku-Rico. $L$ in the equation represents the sample thickness in $\mathrm{cm}$.

$$
\alpha=1.37 L^{2} / \pi^{2} t_{1 / 2}
$$

After the sample was positioned inside, the reaction tube was evacuated and filled with argon gas (AGA, Sweden). Prior to introducing the argon gas to the reaction chamber, the gas was successively passed through columns of silica gel and $\mathrm{Mg}\left(\mathrm{ClO}_{4}\right)_{2}$ (to remove the residual moisture), column of ascarite (to remove $\mathrm{CO}_{2}$ ) and finally through columns of copper and magnesium turnings kept at $923 \mathrm{~K}$ and $723 \mathrm{~K}\left(650^{\circ} \mathrm{C}\right.$ and $450{ }^{\circ} \mathrm{C}$ ), respectively, to remove residual oxygen. ${ }^{[10]}$ Prior to heating, the sample was kept inside the instrument for 36 hours at room temperature under an argon flow of $50 \mathrm{~mL} / \mathrm{min}$. Thermal cycle consisted of heating at the rate of $7 \mathrm{~K} / \mathrm{min}$ to $1423 \mathrm{~K}\left(1150{ }^{\circ} \mathrm{C}\right)$, holding the sample for 270 minutes this temperature, and then cooling it down to the room temperature at the same rate as heating.

Once heating was started, argon flowrate was increased to $200 \mathrm{~mL} / \mathrm{min}$. Further, due to concerns regarding the dissociation of magnetite under very low partial pressures of oxygen, magnesium furnace in the gas cleaning system was turned off. During the whole cycle, thermal diffusivity was monitored every seven minutes. Finally, collected data were corrected with regard to the variation in the base-line as well as the thickness decrease. Selected experiments were repeated in order to confirm the reproducibility of the results.

\section{Thermogravimetric Analysis (TGA)}

TGA experiments were carried out parallely in order to correlate the thermal diffusivity results with mass loss

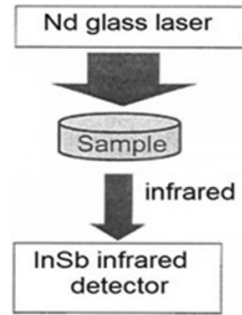

(a)

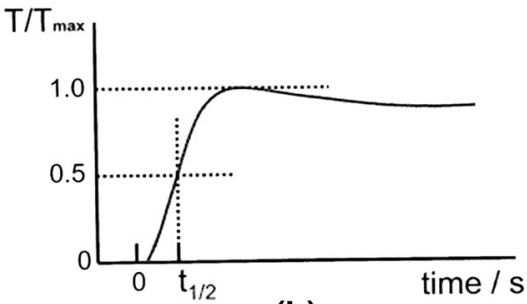

(b)
Fig. 2-(a) laser irradiation on the sample top and temperature response from the back surface and $(b)$ normalized temperature rise vs time. $t=0$ corresponds to the time of impact of the laser shot, reprinted from Ref. [9]. 
occurring during the carbothermic reduction of iron oxide. The results also enabled an estimation of the (apparent) density changes during the progress of the reaction. Samples were compacted into disks with dimensions $7.20 \mathrm{~mm}$ inner diameter and $2.40 \mathrm{~mm}$ in thickness. The experiments were carried out in a SETARAM 92 TGA unit (SETARAM Instrumentation, France). A schematic representation and the experimental details are given in an earlier publication. ${ }^{[1]}$ It is noteworthy that the experimental conditions inside the TGA apparatus were kept the same as the Laser-Flash unit so that the measurements could be synchronized. The results could be confirmed by repetition.

\section{Quenching Studies}

After both thermal diffusivity measurements and TGA, it was observed that the samples showed a remarkable shrinkage. According to the equation for thermal diffusivity (Eq. [8]), sample thickness is an important factor, therefore monitoring dimensional changes proved to be essential when accurate data are desired.

Subsequently, a series of quenching tests were performed to follow the dimensional changes of the sample during the thermal cycle. For this purpose, a horizontal furnace set-up (Figure 3) was employed. Experimental conditions were the same as the Laser-Flash and TGA experiments. Samples (same dimensions as in thermal diffusivity measurements) were quenched at different time intervals by drawing them to the cold end of the tube and cooling them under flushing argon. Thereafter, the final dimensions of the samples were measured at room temperature using a micrometer and thereby, the dimensional changes were calculated. It has to be admitted that this method of following dilatations assumes that thermal expansion is negligible compared to the shrinkage due to sintering.

Collected data were used for the calculation of volume as well as density changes. This was based on the assumption of isotropic shrinkage of the pellet which was confirmed later on by means of a comparison between calculated and measured volumes. Measurement of the apparent volume was based on the buoyancy law and accomplished through an Archimedes test set-up.

\section{E. Metallography and Structure Analysis}

Final product was first studied by X-ray Diffractometer (model D5000, SIEMENS, Germany) in order to characterize the constituent phases. Subsequently, in order to observe the morphology, a series of metallographic investigations were conducted. The sample was cut into half, mounted, and polished. Thereafter, the sample surface was studied by means of SEM. Elemental analysis at different points was carried out through the Energy Dispersive Spectroscopy (EDS). Thereafter, sample was etched by Nital 3 pct solution and studied by a Light Optical Microscope (LOM) aiming at preliminary morphology characterization. Finally, the sample was polished again and this time it was etched by the solution Klemm (I) as cementite was suspected to have formed. Chemical composition and function of the etching solutions are reported in the literature. ${ }^{[12]}$

\section{RESULTS}

\section{A. Characterization of the As-Produced Sample}

According to the SEM micrograph of the initial sample (Figure 4), a uniform distribution of phases could be observed which verifies the effectiveness of performed manual mixing. Average apparent density of the pellets was calculated to be $2.01 \pm 0.03 \mathrm{~g} / \mathrm{cm}^{3}$, which yields a porosity of 53.7 pct. This value was calculated using Eq. [9] (rule of mixture) from the room temperature density of graphite as well as the pure phases in the iron-oxygen binary system. These are summarized in Table I. It has to be mentioned that these are actually averages of the reported values from literature. ${ }^{[2,13]}$

$$
\varepsilon=1-\frac{V_{\text {dense }}}{V_{\text {apparent }}}=1-\frac{\sum_{i=1}^{n}\left(\rho_{i} / m_{i}\right)}{V_{\text {apparent }}},
$$

where $\varepsilon$ is the porosity (pct), $V_{\text {dense, }}$ is the volume of completely dense sample $\left(\mathrm{cm}^{3}\right), V_{\text {apparent }}$ is the measured volume of the sample $\left(\mathrm{cm}^{3}\right), \rho_{i}$ is the density of species $i\left(\mathrm{~g} / \mathrm{cm}^{3}\right)$, and $m_{i}$ is the mass of the same species $(\mathrm{g})$.

\section{B. Measurement of the Effective Thermal Diffusivity}

A maximum deviation of 4 pct from the calibration curve was observed during measurements on the standard sample which was continuously heated/cooled at the rate of $7 \mathrm{~K} / \mathrm{min}$. This confirmed the proper functioning of the instrument and that the interference to the laser energy from the furnace was negligible. These steps ensured that the results obtained are reliable and reproducible.

Results of the measurements on the experimental samples are shown in Figure 5. The line (marked as "raw data") is the raw data obtained if the thickness is assumed constant. As mentioned earlier, in the current case, due to the high temperature of reduction and sintering of the oxides as well as the metallization caused by reduction, considerable decrease in the thickness was found to take place. Accordingly, correction of the acquired data with regard to this effect was necessary. After a series of quenching tests (which will be described in Section IV-D), data on thermal diffusivity were re-processed in order to obtain corrected values. This is shown in Figure 5 by the line marked as "corrected data." It is to be noted from the difference between the two lines that the correction factor is significant.

\section{Thermogravimetry Analysis (TGA)}

The TGA results are shown in Figure 6. Numerical data indicated that the reaction started at about $1173 \mathrm{~K}$ $\left(900{ }^{\circ} \mathrm{C}\right)$. However, the mass loss between $1173 \mathrm{~K}$ and $1273 \mathrm{~K}\left(900{ }^{\circ} \mathrm{C}\right.$ and $\left.1000{ }^{\circ} \mathrm{C}\right)$ was found to be negligible 

1. Gas inlet
2. Cooling water coil
3. Inlet stopper
4. Thermal insulator
5. Alumina tube
6. Heating coils
7. Pressure gauge

8. Gas outlet

9. Thermocouple

10. Outlet stopper

11. Sample holder

12. Sample carrier

13. Rail

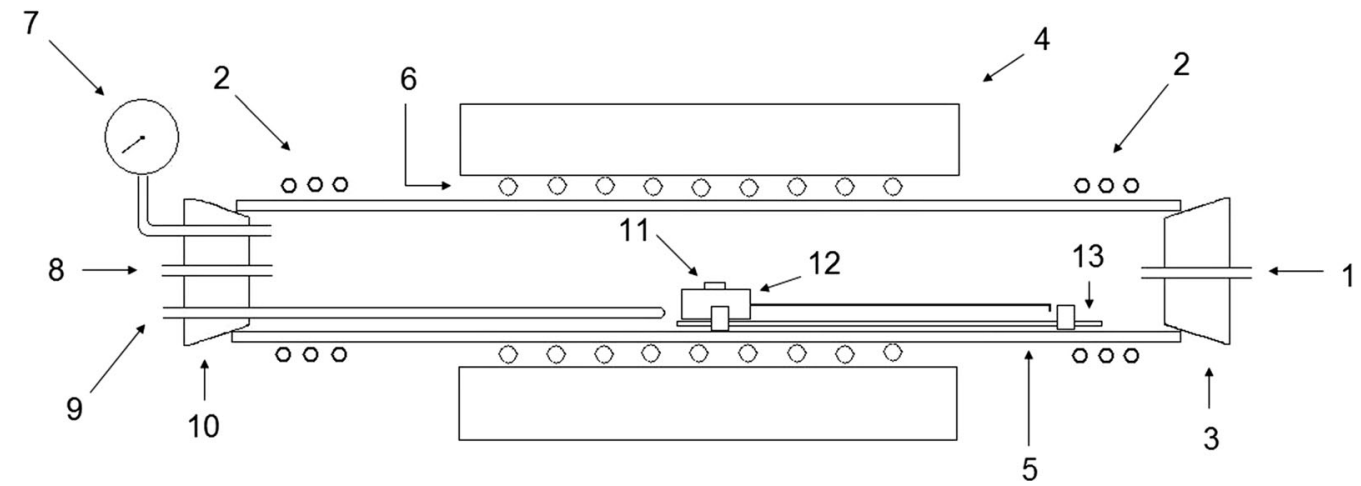

Fig. 3-Horizontal furnace set-up.

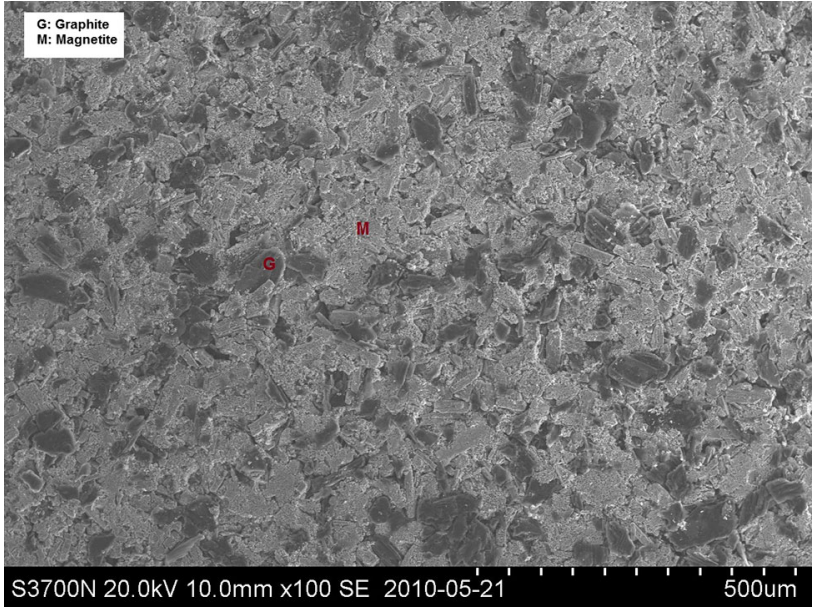

Fig. 4-SEM micrograph (acquired in secondary electron mode) of a composite pellet as starting material.

Table I. Room Temperature Density of Iron, Wüstite, Magnetite, and Magnetite

\begin{tabular}{lcccc}
\hline Phase & $\mathrm{Fe}$ & $\mathrm{FeO}$ & $\mathrm{Fe}_{3} \mathrm{O}_{4}$ & $\mathrm{C}$ \\
\hline Density $\left(\mathrm{g} / \mathrm{cm}^{3}\right)$ & 7.87 & 5.70 & 5.17 & 2.2 \\
\hline
\end{tabular}

(less than 0.1 pct). Significant mass loss could only be observed when the temperature was higher than $1273 \mathrm{~K}$ $\left(1000{ }^{\circ} \mathrm{C}\right)$. Between $1273 \mathrm{~K}$ and $1413 \mathrm{~K}\left(1000{ }^{\circ} \mathrm{C}\right.$ and $1140{ }^{\circ} \mathrm{C}$ ), rate of the reaction was found to increase slowly and an overall mass loss of $\sim 3 \mathrm{wt}$ pet was obtained when the temperature reached $1413 \mathrm{~K}$ $\left(1140{ }^{\circ} \mathrm{C}\right)$. Once the temperature exceeded $1413 \mathrm{~K}$ $\left(1140{ }^{\circ} \mathrm{C}\right)$, rate of the reaction was found to increase suddenly and it quickly proceeded to near-completion. Thereafter, reaction rate suddenly decreased and the rest of the reaction continued somewhat slowly. The overall curve assumed a sigmoidal shape. At the end, a total mass loss of 37.5 pct was achieved which corresponds to about 97 pct reduction degree.

\section{Quenching Tests}

Similar to the TGA curve, thickness changes show a three-stage sigmoidal behavior. Shrinkage (linear change of thickness) was found to start from $\sim 1173 \mathrm{~K}$ $\left(900^{\circ} \mathrm{C}\right)$, but its magnitude was small (less than $1 \mathrm{pct}$ ) up to $1273 \mathrm{~K}\left(1000{ }^{\circ} \mathrm{C}\right)$. Above this temperature, shrinkage rate continuously increased and finally decreased in a way that a total linear shrinkage of 33.7 pct was achieved (Figure 7). Compared to the TGA curve, however, a larger part of the change took place during the isothermal step.

\section{E. Characterization of the Reduced Sample}

Figure 8 shows the SEM micrograph of polished surface of the product. Morphology consists of sponge iron, residual graphite, and considerable amount of porosity. From EDS spot analysis, (points 1 and 2 in the figure), it was revealed that the gray continuous background is the iron phase and the black region on the middle-right part of the image is residual graphite. Rest of the microstructure is porosity as indicated by shiny edges of the iron phase.

Etching the sample by Nital 3 pct and Klemm (I) solutions indicated a ferritic matrix which lacks any pearlite. In view of this, it was concluded that the microstructure consists of iron, residual graphite, and porosity. XRD spectrum of the product revealed that the sample consists mainly of iron and graphite. Amount of magnetite phase was very small and was neglected in further considerations. 


\section{DISCUSSION}

Neglecting the small amount of magnetite in the final product, a total mass loss value of 37.5 pet corresponds to a final composition of $96 \mathrm{wt}$ pet iron and $4 \mathrm{wt}$ pet carbon. Carbothermic reduction of iron oxides is a stepwise process. ${ }^{[8,14]}$ First step corresponds to the conversion of magnetite to wüstite which was observed to occur between $1173 \mathrm{~K}$ and $1413 \mathrm{~K}\left(900{ }^{\circ} \mathrm{C}\right.$ and $\left.1140{ }^{\circ} \mathrm{C}\right)$ during continuous heating at a rate of $7 \mathrm{~K} / \mathrm{min}$. The second step corresponds to the reduction of wüstite to metallic iron and is fast ${ }^{[15]}$ unlike the relatively slow first stage. This is attributed to the autocatalytic effect of iron on Boudouard reaction (gasification of graphite) ${ }^{[16]}$ (Eq. [4]). This effect, in turn, indicates that, before the sharp drop in the TGA curve, no metallic iron has been formed.

A value of 37.5 pct total mass loss suggests that, during the course of the reaction, the off-gas is mainly composed of carbon monoxide. This can be justified considering that a total mass loss of 31.5 pct is achieved if the overall reaction is according to Eq. [10]. If it takes place according to Eq. [11], the mass loss would be 40.1 pet.

$$
\begin{gathered}
\mathrm{Fe}_{3} \mathrm{O}_{4}+2 \mathrm{C}=3 \mathrm{Fe}+2 \mathrm{CO}_{2} \\
\mathrm{Fe}_{3} \mathrm{O}_{4}+4 \mathrm{C}=3 \mathrm{Fe}+4 \mathrm{CO}
\end{gathered}
$$

Since the experiment is performed under inert atmosphere, and proper residual oxygen removal has been done prior to the experiment, the most likely reaction to dominate the reduction initiation is the solid-solid reaction between iron oxide and solid carbon at the contact points (Eq. [2]). Reaction between the two solids, viz., $\mathrm{Fe}_{3} \mathrm{O}_{4}$ and carbon, is expected to have high activation energy. This can be attributed to the separation of a carbon atom and an oxygen atom from the corresponding crystalline lattices and bringing them into an activated complex: This would be more difficult than the formation of an activated complex from a gaseous carbon monoxide molecule and an oxygen atom in the magnetite lattice. This is likely to be the reason for high reaction initiation temperature compared to the reduction of magnetite by a reducing gas. ${ }^{[1]}$

Due to the physicochemical changes that happen as a consequence of the reduction reaction, composite pellet showed a remarkable shrinkage. This is due to the sintering of unreacted oxides and metallic product that take place under the driving force of decrease in the total surface energy of the system. As the pellet has the geometric shape of a disk and its contraction was shown to be isotropic (there was no evidence of any sample distortion), calculation of pellet volume over the whole thermal cycle became possible. Combining the data on sample mass and volume changes, a curve of apparent density $v s$ time can be plotted (Figure 9). During the thermal cycle, both mass and volume would be decreasing. Hence, the rate of decrease in each of these two will determine whether the density would increase or decrease. Calculated curve shows a three step behavior of the density: first increasing, then decreasing, and finally increasing towards saturation. Initially, sintering of oxides dominates the reaction, therefore the density would be expected to increase. Suddenly, second stage of the reaction takes place which accompanies a sudden mass decrease. Since mass decrease dominates the reaction, density decreases rapidly. Immediately after this, sintering of iron starts and density increases until it approaches a value of $4.29 \mathrm{~g} / \mathrm{cm}^{3}$.

At the beginning of the third step, rate of densification is high because the total surface area is high and thereafter as sintering is removing the porosity, the driving force would decrease. Since sintering is not performed in vacuum, densification cannot proceed towards completion as the product gas would be trapped within the closed pores. Difference in the apparent density of the final product and theoretical density of iron can be attributed to this effect.

In Figure 10, the corrected thermal diffusivity curve is re-drawn. For the sake of comparison, the temperature dependencies of thermal diffusivities of pure iron (Akiyama et $a l .{ }^{[2]}$ ) and pure magnetite (Szelagowski [17]) are shown. Difference between the curves can be attributed to the differences in porosity and composition. Porosity of the initial pellet in this study was 53.7 pct, while data from Szelagowski [17] correspond to 24 pet porosity and that from Akiyama et al. ${ }^{[2]}$ to $60 \mathrm{pct}$. In the present work, a value of 40 pct was calculated for the porosity of the product. Therefore, it is considered reasonable that the present values lie between the reported values. Before $t=140$ minutes $[\sim 1273 \mathrm{~K}$ $\left.\left(1000{ }^{\circ} \mathrm{C}\right)\right]$, thermal diffusivity of the composite pellet is following the temperature dependency of the thermal diffusivity of pure magnetite. On the other hand, after $t=430$ minutes (cooling start), thermal diffusivity is following that of pure iron. This is consistent with the data from TGA and apparent density curves. According to these two, no remarkable change is observed below $1273 \mathrm{~K}\left(1000{ }^{\circ} \mathrm{C}\right)$ and after cooling starts.

In Figure 11, thermal diffusivity and TGA data are plotted together. From Figures 9 and 10, two important points regarding the course of physicochemical phenomena during the carbothermic reduction of magnetite can be derived. First point is the decrease in the thermal diffusivity which is coincident with sharp decrease in mass and apparent density ( $t=\sim 160$ minutes). This is due to the conversion of wüstite to metallic iron. This would involve the creation of large amounts of porosity which, in turn, decreases the ability of the pellet to conduct heat through the conduction. Subsequent increase in apparent density and thermal diffusivity ( $t=\sim 170$ to 230 minutes) can be attributed to the sintering of the product as well as higher thermal diffusivity of iron compared to wüstite and magnetite.

After determination of the thermal diffusivity and apparent density changes, the only remaining factor of the thermal conductivity is the heat capacity. In the present work, to estimate the thermal conductivity, heat capacity was calculated based on two assumptions. The 


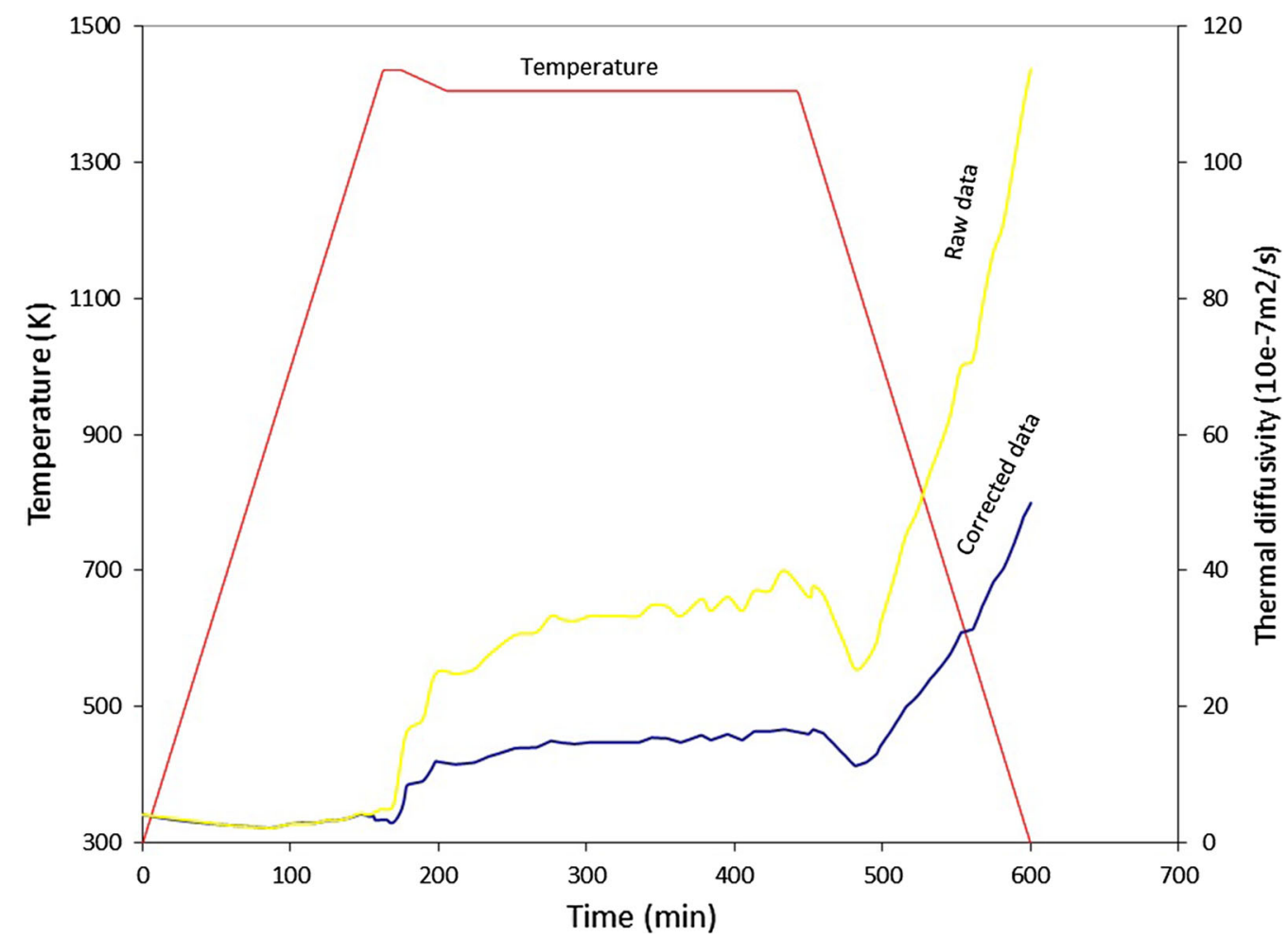

Fig. 5-Effective Thermal diffusivity of the pellet over the whole thermal cycle.

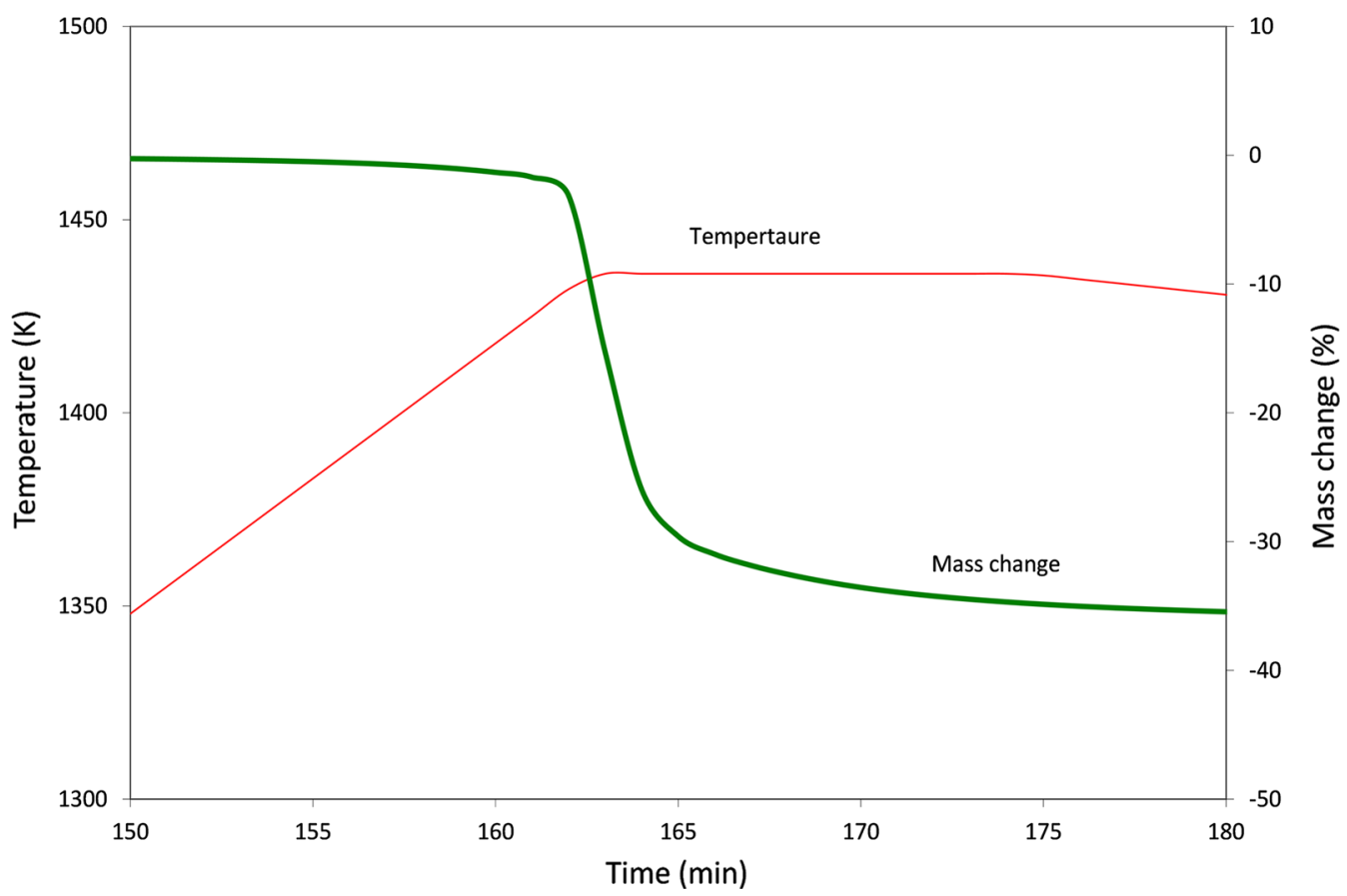

Fig. 6-Mass change of carbon-magnetite composite as a function of time and temperature.

first one was that the reaction is stepwise, in other words from $1173 \mathrm{~K}$ to $1413 \mathrm{~K}\left(900{ }^{\circ} \mathrm{C}\right.$ to $\left.1140{ }^{\circ} \mathrm{C}\right)$, conversion of magnetite to wüstite is taking place and then from
$1413 \mathrm{~K}$ to $1436 \mathrm{~K}\left(1140{ }^{\circ} \mathrm{C}\right.$ to $\left.1163{ }^{\circ} \mathrm{C}\right)$, wüstite is reduced to metallic iron. The second assumption was that during each step, mass fraction of each phase 


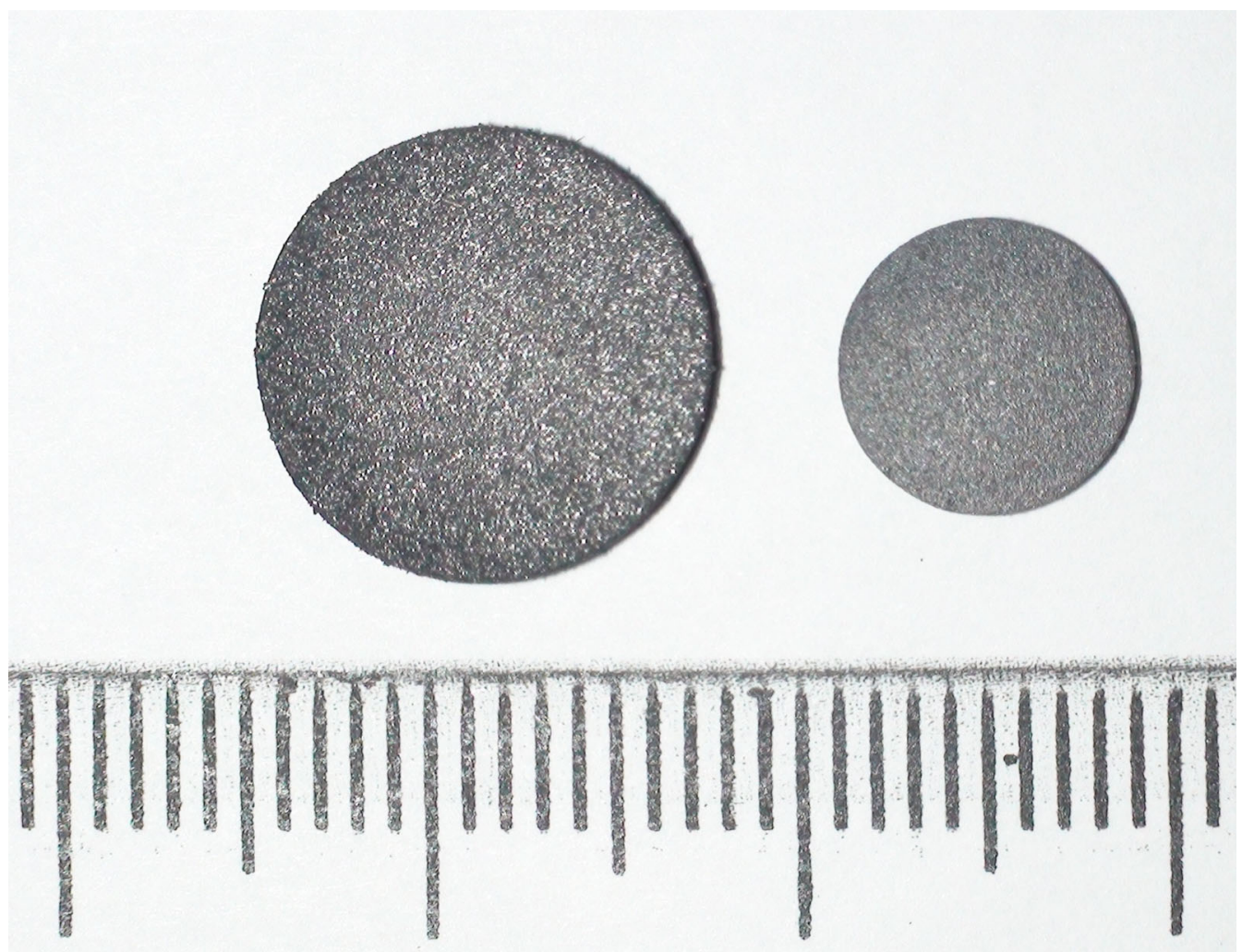

(a)

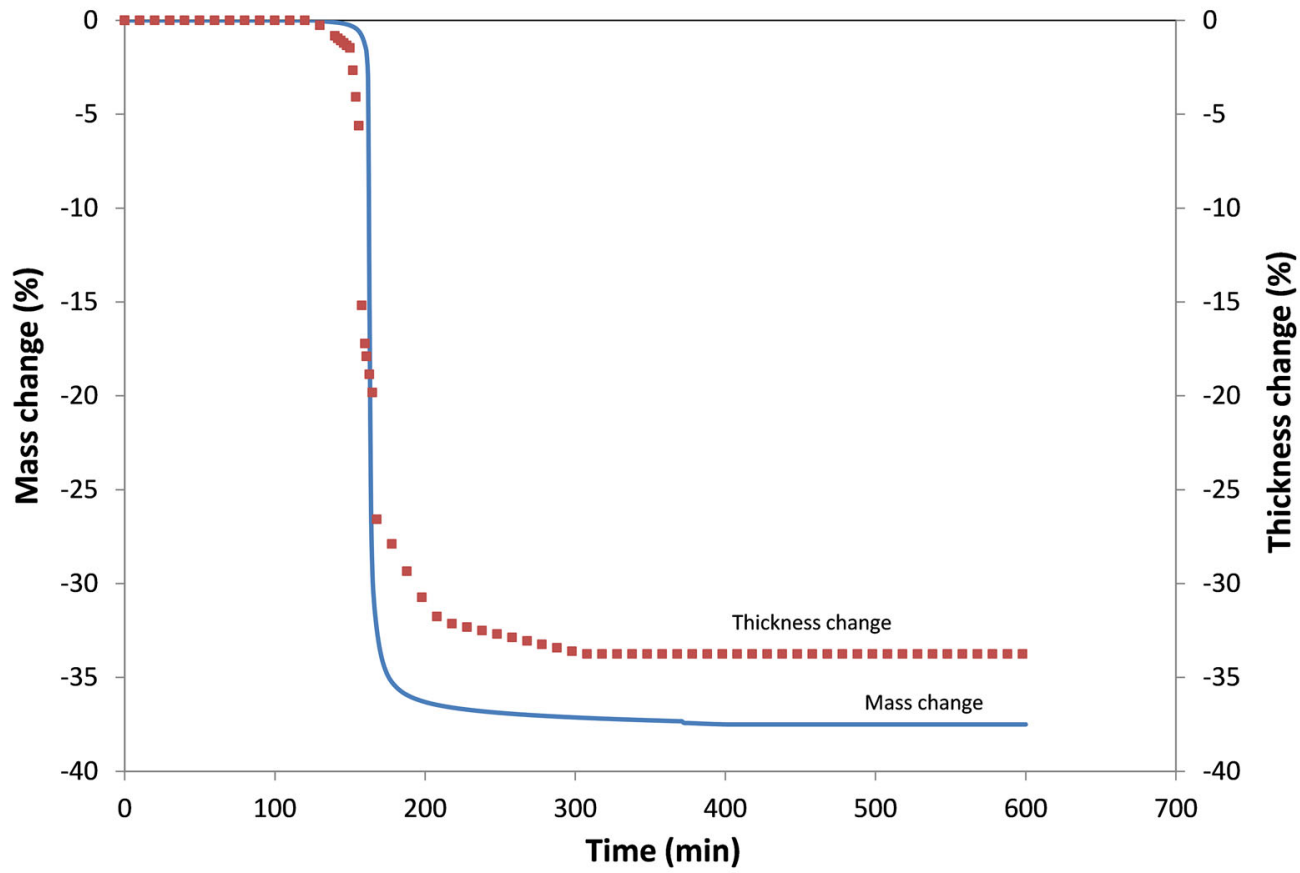

(b)

Fig. 7-(a) Initial pellet (left) and final product (right), major ticks on the ruler designate centimeters and $(b)$ sample thickness change percentage as a function of time and temperature. 
changes linearly with temperature. This was inspired by the work conducted by Liu et al. ${ }^{[14]}$ Subsequently, the total heat capacity of the pellet was calculated by the law of mixtures (Eq. [12]).

$$
C_{\mathrm{p}}=\sum_{i=1}^{n} f_{i} C_{\mathrm{p}, i}
$$

in which $f_{i}$ is the mass fraction and $C_{\mathrm{p}, i}$ is the heat capacity of each phase.

The thermal conductivities estimated as described above are shown in Figure 12. In addition to the current experimental data, separate points represent the experimental data of Akiyama et al. ${ }^{[2]}$ Continuous curves (curves 2 and 5) are the calculated values obtained through a combination of the geometric mean model, ${ }^{[6,18]}$ which accounts for chemical composition

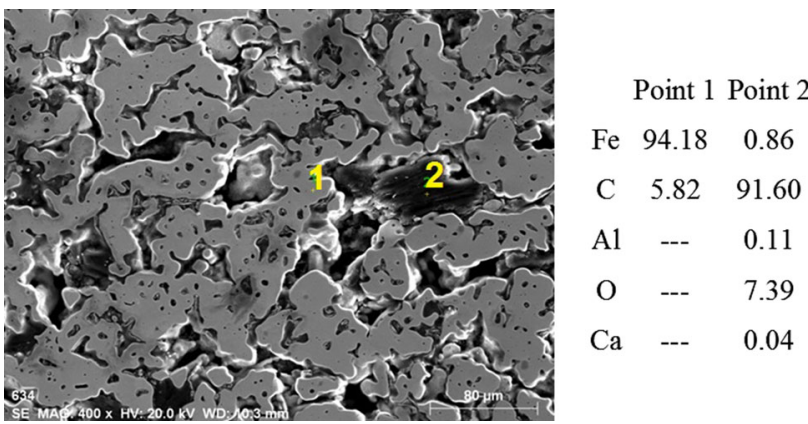

Fig. 8-SEM micrograph of the product (acquired in secondary electron mode) and results of spot analysis performed by EDS. Values in the table are in weight percent. dependency of thermal conductivity, and finite heat transfer model ${ }^{[19]}$ which expresses porosity dependence.

In order to validate and prove the present approach, a comparison between the calculated (based on the present experiment, curve 4) curve and that derived (at corresponding temperatures) from the data reported by Akiyama et al (curves 3,6 and 7) is presented in Figure 12. Due to lack of experimental data for an exactly similar case with regard to composition and porosity, the above comparison can be only qualitative. It shows a similar trend during the heating period which is due to the fact that the initial pellet consists mainly of magnetite. However, since porosity of magnetite pellets in the present case is more than 32 pct, the obtained values lie below those reported by Akiyama et al for fired magnetite. Further, due to the sintering of formed wüstite, the calculated curve lies slightly above the reported values which correspond to 50 pct porosity. ${ }^{[2]}$ Once the second step of the reduction reaction takes place, consistent with sudden creation of large amounts of porosity, thermal conductivity decreases. Thereafter, as sintering of formed iron is taking place, thermal conductivity increases and finally saturates as less and less open pores exist. During cooling, there is significant discrepancy between the present results and the data reported by Akiyama et al. While the present calculated values are higher, this can be justified by the fact that porosity of the produced metallic iron in the present case is less than the reported values of Akiyama et al for the produced metallic iron by $\mathrm{CO}$ reduction (40 pct compared to $62 \mathrm{pct}$ ). Moreover, the present values are more consistent with the trend obtained through modeling calculations for Fe-4 wt pet $\mathrm{C}$ alloy, with 40 pet porosity, as well as experimental values for the temperature dependence of the thermal conductivity of pure dense iron. ${ }^{[2,13]}$

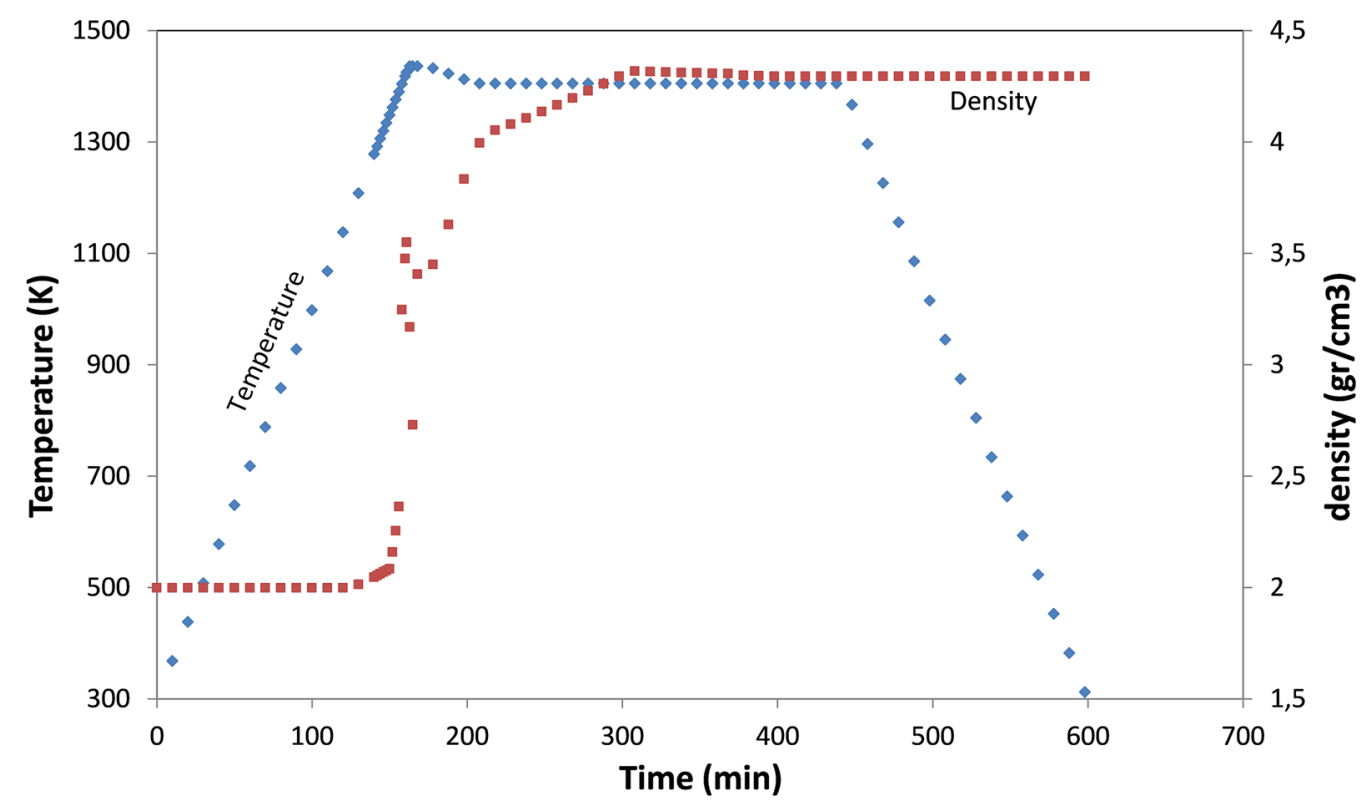

Fig. 9-Sample apparent density as a function of time and temperature. 


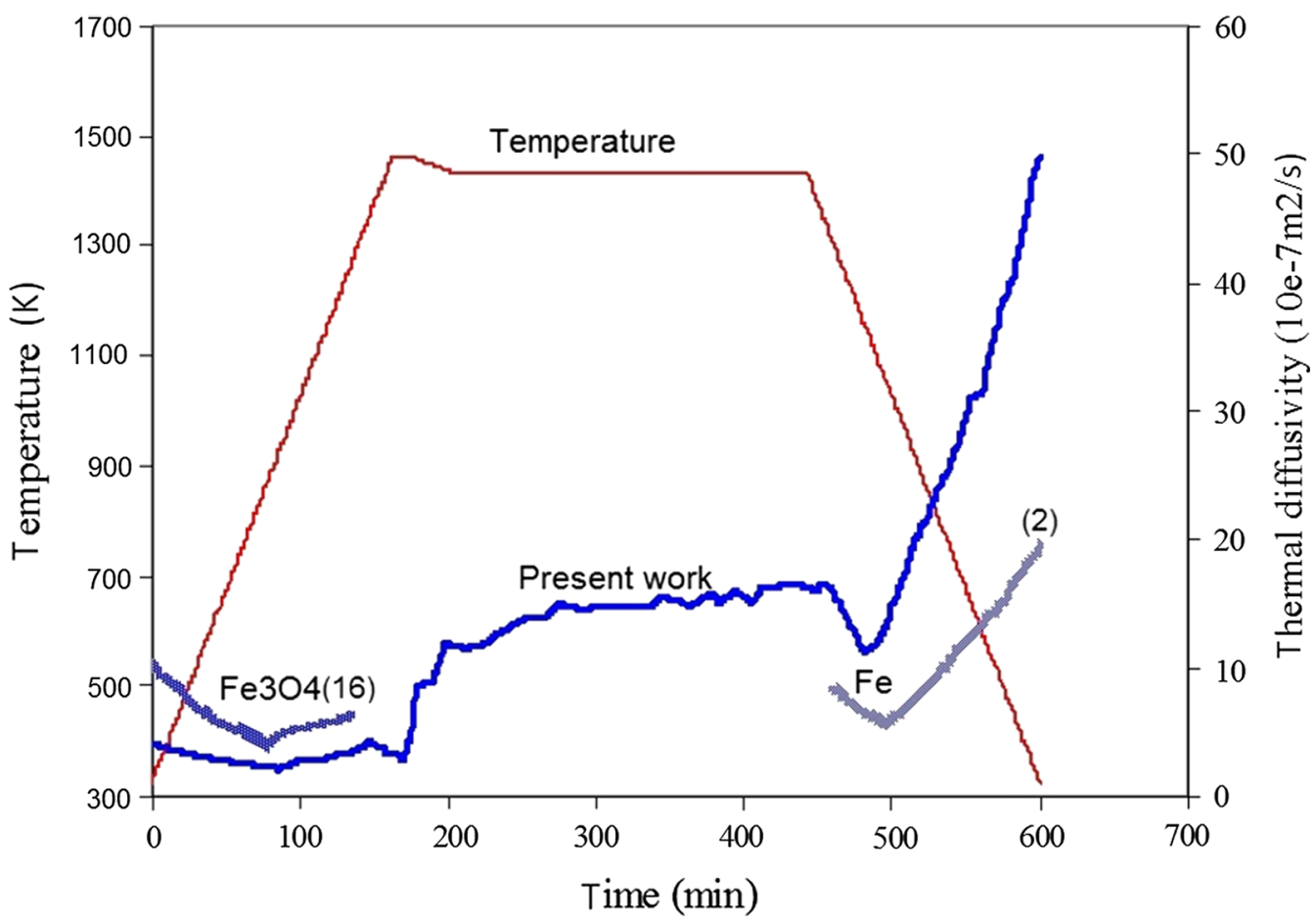

Fig. 10-Comparison of the measured thermal diffusivity with some literature values.

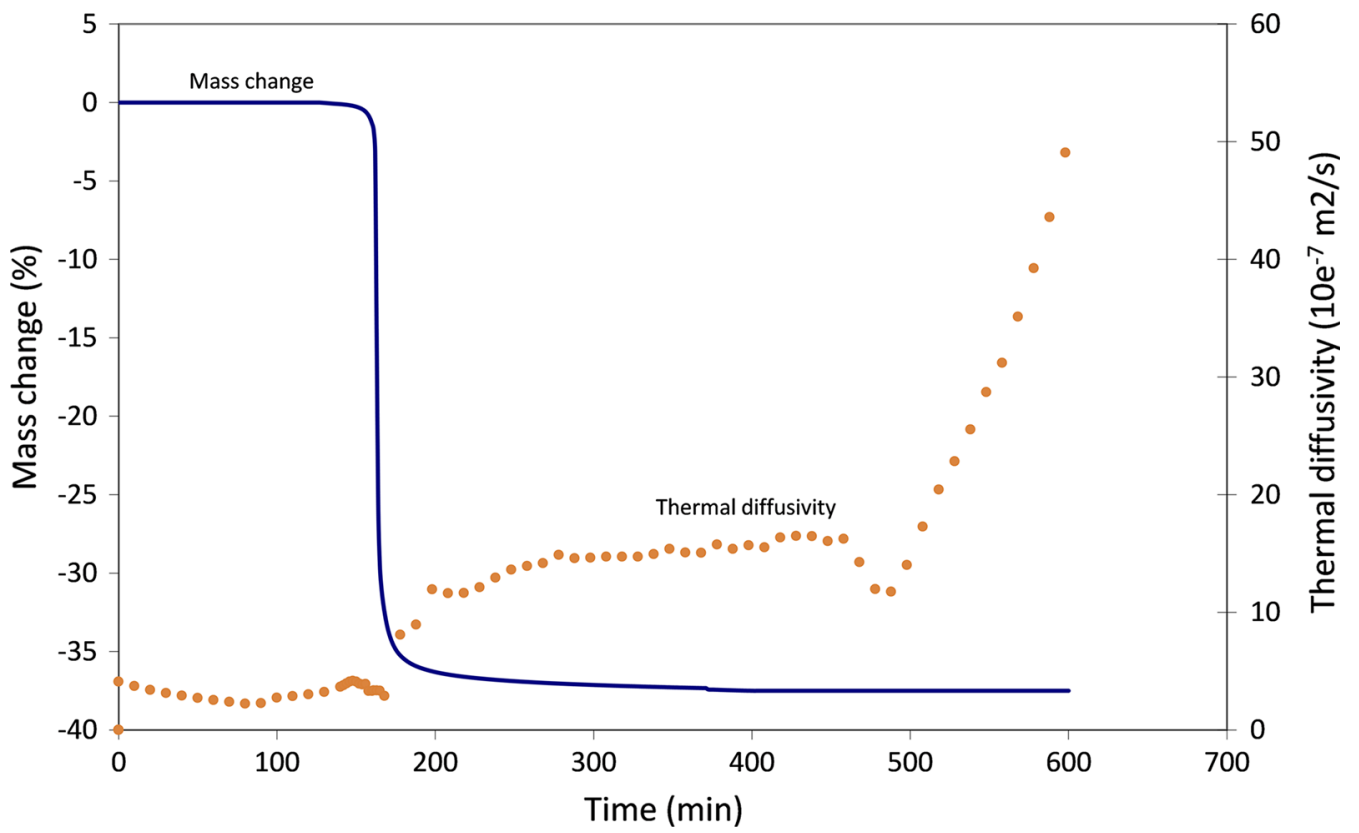

Fig. 11-Thermal diffusivity values as a function of time and mass loss.

\section{SUMMARY}

Effective thermal diffusivity of the magnetite-graphite composite pellet was measured in the dynamic mode by means of laser-flash unit over a pre-defined thermal cycle and under inert atmosphere. It was shown that, for a heating rate of $7 \mathrm{~K} / \mathrm{min}$ or lower, interference of 


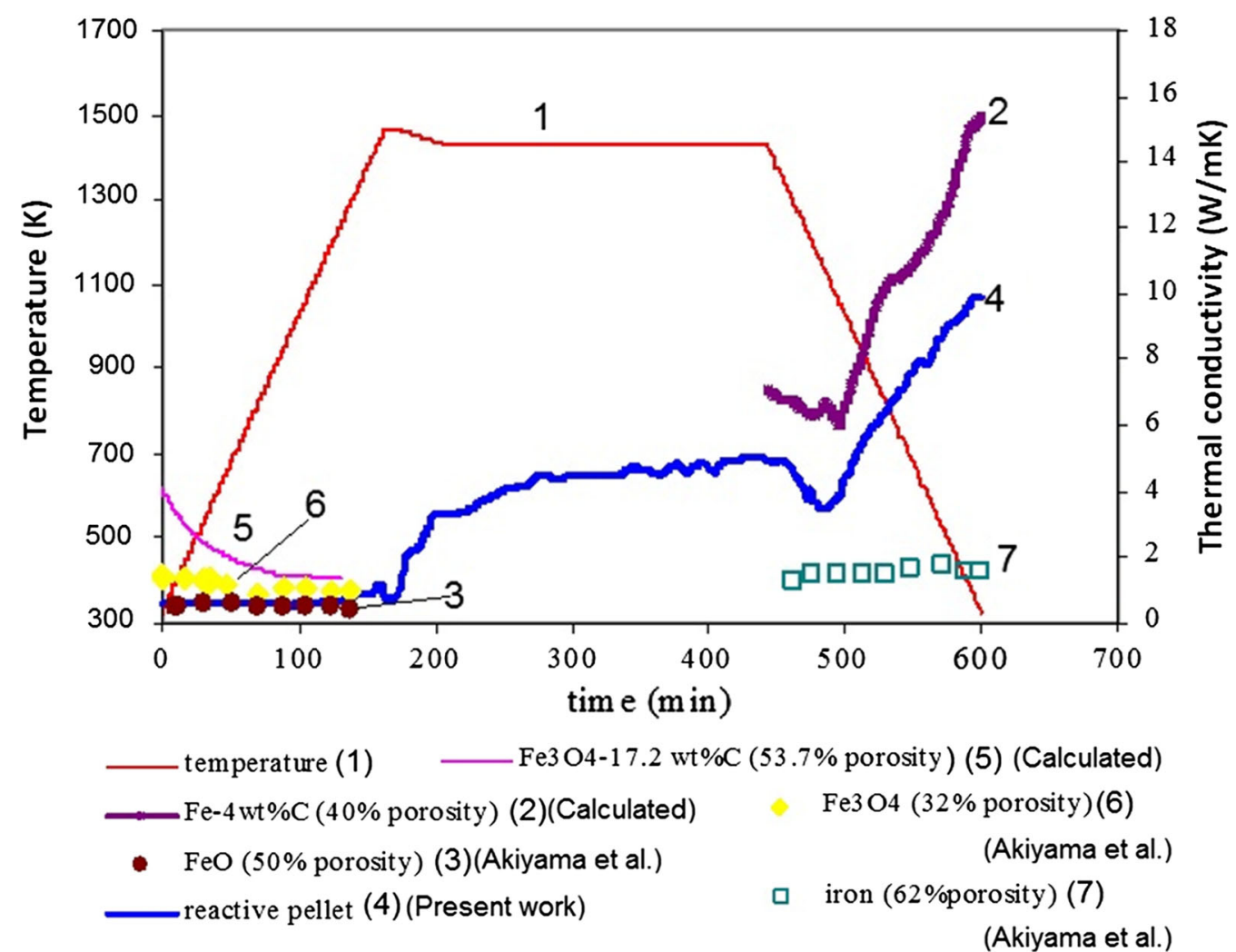

Fig. 12-Estimated changes in thermal conductivity of the reactive magnetite-graphite composite pellet over the whole thermal cycle.

external heat with the laser energy was negligible and dynamic measurements were reliable. Sintering during the course of reaction as well as during the heating of the sample was found to be an important factor which must be considered for sake of accurate dynamic measurements.

Complementary measurements, viz., TGA as well as a series of quenching tests were conducted, under the same experimental conditions as in the thermal diffusivity measurements, to monitor the chemical reaction and volume changes due to sintering. Heat capacity of the pellet was calculated based on some simplifying assumptions. Thus, it became possible to calculate the effective thermal conductivity.

Sintering of oxide, chemical reaction and sintering/carburization of the product are the physicochemical phenomena involved in the reduction of magnetite by graphite. As the chemical reaction led to formation of pores, it decreased the thermal conductivity. In fact, the system showed the lowest thermal diffusivity/conductivity just after the reduction of wüstite to iron. Thereafter, the metallic iron produced started to sinter. Further, as it came into more and more contact with the residual graphite, carburization took place concurrently with the sintering. Just after the reduction of wüstite to iron, rate of increase in the thermal conductivity was found to be high. It then was gradually found to decrease until it finally approached stabilization at a certain value. The stabilization in thermal conductivity value is believed to be due to a drastic decrease in sintering rate. Upon cooling, the reduced samples showed the same temperature dependency of thermal conductivity as in the case of pure iron.

The present investigation indicates the possibility of the in situ measurements of thermal diffusivity and corresponding estimation of the heat capacity, which makes it possible to calculate the continuous change of the thermal conductivity. These values can be further analyzed and modeled to explain the physicochemical changes occurring with the progress of the chemical reaction.

\section{ACKNOWLEDGMENT}

The partial financial support from the Centre of Advanced Mining \& Metallurgy (CAMM), Luleå, Sweden is greatly acknowledged.

\section{OPEN ACCESS}

This article is distributed under the terms of the Creative Commons Attribution 4.0 International License (http://creativecommons.org/licenses/by/4.0/), which permits unrestricted use, distribution, and reproduction in any medium, provided you give appropriate credit to the original author(s) and the source, provide a link to the Creative Commons license, and indicate if changes were made. 


\section{REFERENCES}

1. H.M. Ahmed, N. Viswanathan, and B. Bjorkman: Steel Res. Int., 2014, vol. 85, pp. 293-306.

2. T. Akiyama, H. Ohta, R. Takahashi, Y. Waseda, and J. Yagi: ISIJ Int., 1992, vol. 32, pp. 829-37.

3. P. Chaurasia, D. Chaudhary, and R. Bhandari: J. Appl. Chem. Biotechnol., 1974, vol. 24, pp. 437-45.

4. A.L. Loeb: J. Am. Ceram. Soc., 1954, vol. 37, pp. 96-99.

5. H. Russell: J. Am. Ceram. Soc., 1935, vol. 18, pp. 1-5.

6. W. Woodside and J. Messmer: J. Appl. Phys., 1961, vol. 32, pp. 1688-99.

7. A. Luikov, A. Shashkov, L. Vasiliev, and Y.E. Fraiman: Int. J. Heat Mass Transf., 1968, vol. 11, pp. 117-40.

8. Y. Rao: Chem. Eng. Sci., 1974, vol. 29, pp. 1435-45.

9. R.A. Abas: Experimental Studies of Thermal Diffusivities Concerning some Industrially Important Systems, School of Industrial Engineering and Management, Royal Institute of Technology, 2006.

10. R.A. Abas, M. Hayashi, and S. Seetharaman: Int. J. Thermophys., 2007, vol. 28, pp. 109-22.
11. H.M. Ahmed, A.A. El-Geassy, and S. Seetharaman: Metall. Mater. Trans. B, 2010, vol. 41B, pp. 161-72.

12. G.F. Vander Voort, S.R. Lampman, B.R. Sanders, G.J. Anton, C. Polakowski, J. Kinson, K. Muldoon, S.D. Henry, and W.W. Scott, Jr.: Metallogr. Microstruct., 2004, vol. 9, p. 44073-0002.

13. Y.S. Touloukian and T. Makita: Thermophysical Properties of Matter-The TPRC Data Series. Specific Heat-Nonmetallic Liquids and Gases, Vol. 6S, IFI/Plenum, New Yrok, 1976.

14. G. Liu, V. Strezov, J.A. Lucas, and L.J. Wibberley: Thermochim. Acta, 2004, vol. 410, pp. 133-40.

15. H.M. Ahmed, A. Persson, L.S. Okvist, and B. Bjorkman: ISIJ Int., 2015, vol. 55, pp. 2082-89.

16. N. Srinivasan and A. Lahiri: Metall. Trans. B, 1977, vol. 8, pp. 175-78.

17. H. Szelagowski: Powder Technology Project Report, 1998.

18. L. Marcussen: Mathematical Models for Effective Thermal Conductivity, Springer, New York, 1985, pp. 585-98.

19. M. Hayashi, R. Rajter, R. Morales, and S. Seetharaman: Z. Metall., 2003, vol. 94, pp. 1179-84. 